Conclusions RC-time is not constant between health and pulmonary vascular disease. A reduction in RC-time, in the context of $\mathrm{PAH}$, is associated with a decrease in cardiac efficiency. RVOPF is lower in the NPH group compared to the PAH group. This implies better cardiac efficiency in the NPH group possibly due to less pulsatile loading of the RV. Haemodynamic assessments which include measures of compliance may be of utility in understanding the progression of right heart failure in $\mathrm{PAH}$.

\section{P172 PULMONARY HYPERTENSION IN IPF: UTILITY OF HRCT}

${ }^{1} \mathrm{G}$ Bettini, ${ }^{1} \mathrm{MA}$ Mazzei, ${ }^{1} \mathrm{D}$ Castria, ${ }^{1} \mathrm{E}$ Kacerja, ${ }^{1} \mathrm{RM}$ Refini, ${ }^{2} \mathrm{~F}$ De Negri, ${ }^{1} \mathrm{~N}$ Cioffi Squitieri, 'S Guerrini, 'FG Mazzei, 'P Rottoli, 'L Volterrani. 'Department of Medical, Surgical and Neuro Sciences, University of Siena, Italy., Siena, Italy; ${ }^{2}$ Department of Emergency Medicine, University of Pisa, Pisa, Italy

\subsection{6/thoraxjnl-2014-206260.301}

Purpose To evaluate the reliability of HRCT-parameters suggesting Pulmonary Hipertension $(\mathrm{PH})$ in a population of Idiopathic Pulmonary Fibrosis (IPF) patients with or without $\mathrm{PH}$ at rightsided-heart-catheterization.

Methods and materials The HRCT scans of 26 patients with IPF, underwent both right-sided-heart-catheterization and echocardiography, were retrospectively, blindly and independently, evaluated by one radiology resident and one experienced-radiologist (with a 10-years-experience in thoracic-radiology).

The diameter and area of Pulmonary Artery (PA), before its bifurcation, the diameter of ascending aorta and the mid anteroposterior-diameter of the thoracic vertebra, on the same CTsection, and the widest short-axis-diameters of four segmental arteries and bronchus, in both upper and lower lobes, were measured. The diameters of left ventricle and inferior vena cava, the presence of emphisema, pericardial effusion and hiatal hernia were also evaluated.

Results 15 out of 29 patients had PH at RHC. The HRCT parameters related to the mean pulmonary artery pressure (mPAP) were: the PA-area $\left(\mathrm{R}^{2}=0.238079 ; \mathrm{p}=0.04\right)$, the ratio of the PA-area to the ascending aorta diameter $\left(\mathrm{R}^{2}=0.215345\right.$; $\mathrm{p}$ $=0.0524)$ and above all the ratio of the diameter of segmental artery to the adjacent bronchus in the left upper lobe $\left(\mathrm{R}^{2}=0.354973 ; \mathrm{p}=0.0006\right)$.

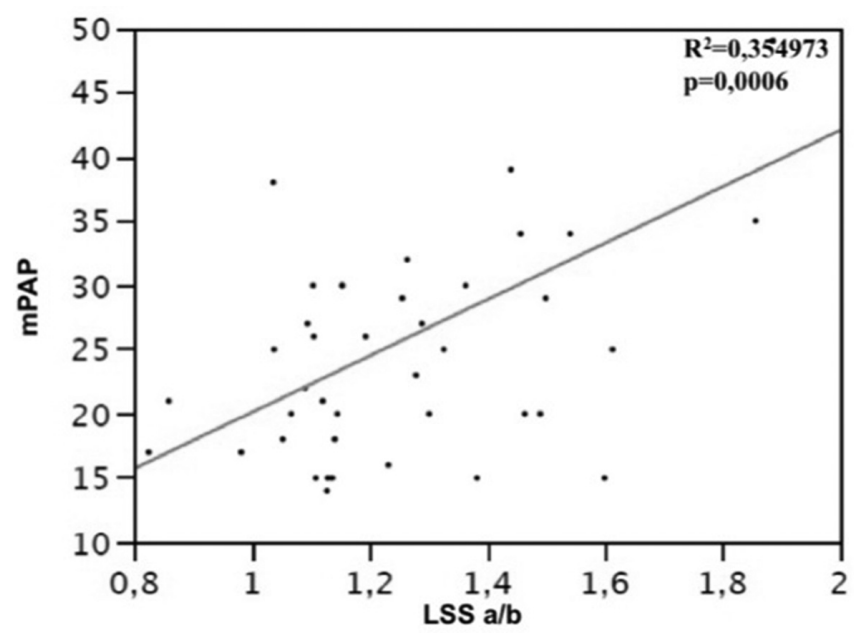

Abstract P172 Figure 1 Linear regression between mean puhnonary artery pressure measured by right heart catheterization (mPAP) and the ratio of the diameter of segmental artery to the adjacent bronchus of the apicoposterior segment of the left upper lobe
The combination of systolic pulmonary artery pressure (sPAP), the PA-area at HRCT and the ratio of the diameter of segmental artery to the adjacent bronchus in the apicoposterior segment of the left upper lobe was strongly correlated to mPAP $\left(\mathrm{R}^{2}=0.785163 ; \mathrm{p}=0.0001\right)$. The contribution of other echocardiographic-parameters (longitudinal STRAIN and Time-to-Peak STRAIN values, TTP) in multivariate regression analysis was not statistically significant, probably because of the small number of patients.

Using the ROC Analysis we found that 931,6 is the upper limit of normal (ULN) for the PA-area, with a $86 \%$ sensitivity and $61 \%$ specificity $(0.839$ AUC); while 20.34 is the ULN for the ratio of the PA-area to the ascending aorta diameter, with a 100\% sensitivity and 50\% specificity (0.804 AUC).

Conclusion (S) HRCT remains an useful tool to identify patients with $\mathrm{PH}$, however the combination of HRCT and echocardiograpy improves accuracy in PH diagnosis.

\section{In the pleural zone}

\section{P173 AMBULATORY MANAGEMENT OF SPONTANEOUS PNEUMOTHORAX}

K Thomas, M Naeem, RV Reddy. Kettering General Hospital, Kettering, UK

\subsection{6/thoraxjnl-2014-206260.302}

Introduction There is no clear consensus on the management of Spontaneous pneumothorax. BTS recommends insertion of chest drain following failure of initial aspiration in large primary spontaneous pneumothorax (PSP) and in all patients with symptomatic/ large secondary spontaneous pneumothorax (SSP). These patients are usually admitted to hospital following chest drain insertion.

Objective To study the feasibility and safety of early discharge of spontaneous pneumothorax patients requiring chest drain on the ambulatory pathway with a Heimlich valve (pneumostat device).

Methods Patients were initially managed as per BTS guidelines. Patients who had a chest drain inserted were admitted until review by the respiratory team. All PSP patients and some SSP patients with good performance status (WHO scale 0-1) were eligible for the ambulatory pathway. Those with continuing air leak are fitted with a Heimlich valve and discharged home. They were reassessed every two days with a CXR on arrival in the ambulatory care unit. The chest drain was removed once the air leak stopped for at least $24 \mathrm{~h}$.

Results 21 episodes of spontaneous pneumothorax in 18 patients (10 PSP and 8 SSP) were treated on the ambulatory pathway between May'13 and June'14. The healthcare usage of patients on ambulatory pathway is listed in the table. The pneumothorax resolved successfully in $82 \%$ (17 episodes). There were three recurrences requiring repeat management on the ambulatory pathway. A total of 10 patients were referred to the surgeon including four with continuing air leak and six due to

\begin{tabular}{lll} 
Abstract P173 Table 1 & & \\
\hline & Range & Average \\
\hline Duration in hospital (days) & $0-4$ & 1.62 \\
Duration in community (days) & $1-12$ & 3.28 \\
Number of reviews & $1-4$ & 1.57 \\
\hline
\end{tabular}


recurrence. Patients with continuing air leak remained in the community until admission for thoracic surgery. Complications included pain in two patients and allergic reaction to the dressing used in one patient. The patient with allergic reaction had accidental dislodgement of chest drain during dressing change necessitating reinsertion of chest drain.

Conclusion It is feasible for most patients with large PSP and many patients with SSP to be managed on an ambulatory care pathway with a Heimlich valve until their pneumothorax heals or is definitively treated.

\section{REFERENCE}

1 Fanny Voisin et al. Ambulatory management of spontaneous pneumothorax with pigtail catheters. http://dx.doi.org. 10.1016/j.annemergmed.2013.12.017

\section{P174 UTILITY OF NEEDLE ASPIRATION IN PATIENTS WITH PRIMARY SPONTANEOUS PNEUMOTHORAX WITH COMPLETE LUNG COLLAPSE: A RETROSPECTIVE 5-YEAR STUDY}

MB Ganaie, S Bikmalla, MA Afridi, MA Khalil, IR Hussain, M Haris. University Hospital of North Staffordshire, Stoke on Trent, UK

\subsection{6/thoraxjnl-2014-206260.303}

Introduction Primary spontaneous pneumothorax (PSP) is a common presentation. Despite being known for over 200 years, there is variation in definition criteria and treatment recommendations. Previous studies comparing needle aspiration (NA) with intercostal drain (ICD) for all primary spontaneous pneumothoraces requiring intervention including those with complete lung collapse have shown no difference in immediate success, early failure and recurrence rate. There is no separate treatment algorithm for PSP with complete lung collapse in the current British Thoracic Society pneumothorax guidelines. We aimed to compare NA with ICD as the first intervention in this subgroup.

Methods We conducted a retrospective observational study of 735 consecutive pneumothorax episodes between March 2008 and December 2013. Those with secondary spontaneous pneumothorax, history of trauma and iatrogenic pneumothorax were excluded. Pneumothorax with no visible aerated ipsilateral lung on plain chest radiograph was defined as 'PSP with complete lung collapse'. Patient case records and plain chest radiographs were reviewed. Values of $\mathrm{p}<0.05$ were considered statistically significant.

Results Of the 735 episodes, 233 (32\%) were PSP. 61 PSP patients were identified to have complete lung collapse on chest radiograph. 32 patients had NA and 29 ICD as the first intervention. There was no statistically significant difference between the two groups in terms of age, sex, smoking history and symptoms. Compared to the NA group, patients with ICD had significantly better immediate success rate $(66 \%$ vs. $10 \% ; \mathrm{p}<0.0001)$ and lower rate of recurrence ( $3 \%$ vs. $31 \% ; p=0.0064)$. Median length of stay was similar in both groups. Almost a third of the patients in both groups required a definitive surgical intervention.

Conclusion Our results suggest significantly better success with ICD as the first intervention in the management of PSP with complete lung collapse and there was no added benefit of NA. We propose a further sub group of PSP with complete lung collapse in which NA should not be attempted.

\begin{tabular}{|c|c|c|c|}
\hline & $\begin{array}{l}\text { NA as first } \\
\text { intervention } \\
(n=32)\end{array}$ & $\begin{array}{l}\text { ICD as first } \\
\text { intervention } \\
(n=29)\end{array}$ & $P$ value \\
\hline Median age, years (IQR) & $31(25-39)$ & $32(26-36)$ & 0.66 \\
\hline Male, n (\%) & $21(66 \%)$ & $22(76 \%)$ & 0.41 \\
\hline Right sided, n (\%) & $19(59 \%)$ & $18(62 \%)$ & $>0.99$ \\
\hline Previous pneumothorax, n (\%) & $4(13 \%)$ & $5(17 \%)$ & 0.72 \\
\hline \multicolumn{4}{|l|}{ Smoking history } \\
\hline Current/Ex, n (\%) & $26(82 \%)$ & $21(75 \%)$ & 0.54 \\
\hline Never, $\mathrm{n}(\%)$ & $3(9 \%)$ & $6(21 \%)$ & 0.29 \\
\hline Mean pack years, n (IQR) & $9(5-20)$ & $12.5(8.5-20)$ & 0.49 \\
\hline \multicolumn{4}{|l|}{ Symptoms } \\
\hline Chest pain, n (\%) & $26(81 \%)$ & $24(83 \%)$ & $>0.99$ \\
\hline Dyspnoea, n (\%) & $27(84 \%)$ & $28(97 \%)$ & 0.20 \\
\hline Cough, n (\%) & $4(13 \%)$ & $8(28 \%)$ & 0.20 \\
\hline Median LOS, days (IQR) & $6(4-10)$ & $9(4-13)$ & 0.19 \\
\hline Successful lung re-expansion, $\mathrm{n}(\%)$ & $3(10 \%)$ & $19(66 \%)$ & $<0.0001$ \\
\hline \multicolumn{4}{|l|}{ Need for surgical intervention, $\mathrm{n}$} \\
\hline$(\%)$ & $10(31 \%)$ & $10(34 \%)$ & $>0.99$ \\
\hline Recurrence, $\mathrm{n}(\%)$ & $10(31 \%)$ & $1(3 \%)$ & 0.0064 \\
\hline
\end{tabular}

\section{P175 MEASUREMENT OF AIR LEAK POST-THORACIC SURGERY: IMPLICATIONS FOR MEDICAL MANAGEMENT OF PNEUMOTHORAX}

${ }^{1} \mathrm{RJ}$ Hallifax, ${ }^{2} \mathrm{~J}$ Mitchell, ${ }^{1} \mathrm{JP}$ Corcoran, ${ }^{1} \mathrm{I}$ Psallidas, ${ }^{1} \mathrm{NM}$ Rahman, ${ }^{1} \mathrm{E}$ Belcher. ${ }^{1}$ Oxford Centre for Respiratory Medicine, Oxford, UK; ${ }^{2}$ Department of Thoracic Surgery, Oxford, UK

\subsection{6/thoraxjnl-2014-206260.304}

Introduction Use of digital suction devices post-thoracic surgery is widespread, allowing patients to be more mobile and potentially reducing the time to chest drain removal post-op (in comparison with standard underwater seal).

Spontaneous pneumothorax (SP) is common $(5,000 / \mathrm{yr}$ in the UK). However, there are no good predictors of outcome for patients with pneumothorax. Measurement of early air leak could potentially predict which patients who will not resolve spontaneously and will require surgery. Post-surgical data may provide an interesting analogy to ongoing air leak in spontaneous pneumothorax. The hypothesis is that reduction in air leak to $<50 \mathrm{ml} / \mathrm{min}$ within $30 \mathrm{~min}$ of attachment can predict air leak over next $48 \mathrm{~h}$ and overall drain duration.

Methods Retrospective review of the use of digital suction device (Thopaz, Medela UK) post-op in the Thoracic Surgical department of a tertiary referral centre between May and December 2012. The detailed air leak measurements were assessed against duration of drainage.

Results Operations included 88 lung resections (wedge resections, lobectomies and metastasectomies via VATS and thoracotomy), 28 pleural procedures (VATS pleurodesis +/- bullectomy) and 12 empyema drainage/decortication. Average air leak over the entire duration was significantly different between the groups: $80.6 \mathrm{ml} /$ $\min , 54.3 \mathrm{ml} / \mathrm{min}$ and $304.5 \mathrm{ml} / \mathrm{min}$ respectively $(\mathrm{p}=0.01)$.

Patients with early reduction of air leak (i.e. reduced to $<50$ $\mathrm{ml} / \mathrm{min}$ within the initial 30 mins) were compared to patients 